\title{
Search for Neutrinoless Double Beta Decay with the GERDA experiment: Phase II
}

\author{
M. Agostini ${ }^{a}$, M. Allardt ${ }^{d}$, A.M. Bakalyarov ${ }^{m}$, M. Balata ${ }^{a}$, I. Barabanov ${ }^{k}$, L. Baudis ${ }^{s}$, C. Bauer ${ }^{g}$, \\ E. Bellotti ${ }^{h, i}$, S. Belogurov ${ }^{l, k}$, S.T. Belyaev ${ }^{m}$, G. Benato ${ }^{s}$, A. Bettini ${ }^{p, q}$, L. Bezrukov ${ }^{k}$, T. Bode ${ }^{o}$, \\ D. Borowicz ${ }^{c, e}$, V. Brudanin $e$, R. Brugnera ${ }^{p, q}$, A. Caldwell ${ }^{n}$, C. Cattadori ${ }^{i}$, A. $_{\text {Chernogorov }} l$, \\ V. D’Andrea ${ }^{a}$, E.V. Demidova ${ }^{l}$, N. Di Marco ${ }^{a}$, A. Domula ${ }^{d}$, E. Doroshkevich ${ }^{k}$, V. Egorov $^{e}$, \\ R. Falkenstein ${ }^{r}$, N. Frodyma ${ }^{c}$, A. Gangapshev $k, g$, A. Garfagnini $p, q$, C. Gooch ${ }^{n}$, P. Grabmayr ${ }^{r}$, \\ V. Gurentsov ${ }^{k}$, K. Gusev ${ }^{e, m, o}$, J. Hakenmüller ${ }^{g}$, A. Hegai ${ }^{r}$, M. Heisel ${ }^{g}$, S. Hemmer ${ }^{q}$, W. Hofmann ${ }^{g}$, \\ M. Hult ${ }^{f}$, L.V. Inzhechik ${ }^{k}$, J. Janicskó Csáthy ${ }^{* o}$, J. Jochum ${ }^{r}$, M. Junker ${ }^{a}$, V. Kazalov ${ }^{k}$, T. Kihm $^{g}$, \\ I.V. Kirpichnikov ${ }^{l}$, A. Kirsch ${ }^{g}$, A. Kish ${ }^{s}$, A. Klimenko ${ }^{g, e}$, R. KneißI ${ }^{n}$, K.T. Knöpfle ${ }^{g}$, O. Kochetov ${ }^{e}$, \\ V.N. Kornoukhov ${ }^{l, k}$, V.V. Kuzminov ${ }^{k}$, M. Laubenstein ${ }^{a}$, A. Lazzaro ${ }^{o}$, V.I. Lebedev ${ }^{m}$, B. Lehnert ${ }^{d}$, \\ H.Y. Liao ${ }^{n}$, M. Lindner ${ }^{g}$, I. Lippi ${ }^{q}$, A. Lubashevskiy ${ }^{g, e}$, B. Lubsandorzhiev ${ }^{k}$, G. Lutter ${ }^{f}$, \\ C. Macolino ${ }^{a}$, B. Majorovits ${ }^{n}$, W. Maneschg ${ }^{g}$, E. Medinaceli ${ }^{p, q}$, M. Miloradovic ${ }^{s}$, R. Mingazheva ${ }^{s}$, \\ M. Misiaszek ${ }^{c}$, P. Moseev ${ }^{k}$, I. Nemchenok ${ }^{e}$, D. Palioselitis ${ }^{n}$, K. Panas ${ }^{c}$, L. Pandola ${ }^{b}$, K. Pelczar $^{c}$, \\ A. Pullia ${ }^{j}$, S. Riboldi ${ }^{j}$, N. Rumyantseva ${ }^{e}$, C. Sada ${ }^{p, q}$, F. Salamida ${ }^{i}$, M. Salathe ${ }^{g}$, C. Schmitt $^{r}$, \\ B. Schneider ${ }^{d}$, S. Schönert ${ }^{o}$, J. Schreiner ${ }^{g}$, O. Schulz ${ }^{n}$, A.-K. Schütz ${ }^{r}$, B. Schwingenheuer ${ }^{g}$, \\ O. Selivanenko ${ }^{k}$, E. Shevchik ${ }^{e}$, M. Shirchenko ${ }^{e}$, H. Simgen ${ }^{g}$, A. Smolnikov ${ }^{g, e}$, L. Stanco $^{q}$, \\ L. Vanhoefer ${ }^{n}$, A.A. Vasenko ${ }^{l}$, A. Veresnikova ${ }^{k}$, K. von Sturm ${ }^{p, q}$, V. Wagner ${ }^{g}$, A. Wegmann ${ }^{g}$, \\ T. Wester $^{d}$, C. Wiesinger $\left.{ }^{*}\right)$, M. Wojcik ${ }^{c}$, E. Yanovich ${ }^{k}$, I. Zhitnikov ${ }^{e}$, S.V. Zhukov ${ }^{m}$, D. Zinatulina ${ }^{e}$, \\ K. Zuber ${ }^{d}$, G. Zuzel ${ }^{c}$. \\ $\left.{ }^{a}\right)$ LNGS \& GSSI (INFN), ${ }^{b}$ ) LNS (INFN), ${ }^{c}$ ) IoP Cracow, ${ }^{d}$ ) TU Dresden, ${ }^{e}$ ) JINR Dubna, ${ }^{f}$ ) EU, Geel, \\ ${ }^{g}$ ) MPI Heidelberg, ${ }^{h}$ ) U. Bicocca Milan, ${ }^{i}$ ) INFN Bicocca Milan, ${ }^{j}$ ) U.d.Studi Milan, ${ }^{k}$ ) INR Moscow, \\ ${ }^{l}$ ) ITEP Moscow, ${ }^{m}$ ) Kurchatov Moscow, ${ }^{n}$ ) MPI Munich, ${ }^{o}$ ) TUM Munich, ${ }^{p}$ ) U. Padova, ${ }^{q}$ ) INFN Padova, \\ ${ }^{r}$ ) EKUT Tübingen, ${ }^{s}$ ) U. Zurich \\ E-mail: gerda-eb@mpi-hd.mpg.de
}

The GERmanium Detector Array (GERDA) experiment, located at the Gran Sasso underground laboratory in Italy, is built for the search of $0 v \beta \beta$ decay in ${ }^{76} \mathrm{Ge}$. GERDA operates bare high purity germanium detectors submersed in liquid Argon (LAr). Phase I of the experiment was completed reaching an exposure of about $21 \mathrm{~kg} \cdot \mathrm{yr}$ with a background level of $10^{-2} \mathrm{cts} /(\mathrm{keV} \cdot \mathrm{kg} \cdot \mathrm{yr})$. GERDA Phase I set a limit on the $0 v \beta \beta$ decay of ${ }^{76} \mathrm{Ge}$ of $T_{1 / 2}^{0 v}>2.1 \cdot 10^{25} \mathrm{yr}$. In Phase II $35 \mathrm{~kg}$ of germanium detectors enriched in ${ }^{76} \mathrm{Ge}$ are operated to reach an exposure of $100 \mathrm{~kg} \cdot \mathrm{yr}$. The design goal is to reduce the background by one order of magnitude to reach the sensitivity for $T_{1 / 2}^{0 v}=\mathscr{O}\left(10^{26}\right)$ yr. The Phase II setup comprises thirty newly produced Broad Energy Germanium (BEGe) detectors. They contribute to the background reduction with better energy resolution and enhanced pulse shape discrimination. To achieve the necessary background reduction, the setup was complemented with LAr veto. The hardware upgrade for Phase II was finished and all detectors were deployed in December 2015. We present the first results of Phase II with $10.8 \mathrm{~kg} \cdot \mathrm{yr}$ exposure reached in June 2016.

38th International Conference on High Energy Physics

3-10 August 2016

Chicago, USA

* Speaker. 


\section{The Gerda Experiment}

A potential observation of the $0 v \beta \beta$ decay would be a measurement of the effective neutrino mass. By setting limits on the effective neutrino mass with the next generation experiments we will be probing the mass ordering of neutrinos accessing a parameter range that implies inverted hierarchy. The predictions of left-right symmetric models can be already tested with existing and next generation experiments [1]. Existing and future $0 v \beta \beta$ experiments are also testing models involving right handed currents and heavy neutral leptons. The parameter space covered by $0 v \beta \beta$ experiments overlaps with the ones probed by LHC and future accelerator experiments [2].

The GERmanium Detector Array (GERDA) experiment is using high-purity germanium detectors (HPGe) for the search of $0 v \beta \beta$ decay. The HPGe detectors are made of germanium enriched in the ${ }^{76} \mathrm{Ge}$ isotope and they are serving as source and detector in the same time.

A big advantage of using HPGe detectors for the $0 v \beta \beta$ decay search consists in the fact that due to the excellent energy resolution a distinct peak should appear at the $Q$ value of the decay if $0 v \beta \beta$ decay exists. As a consequence the $2 v \beta \beta$ decay does not constitute a background for $0 v \beta \beta$ decay. Furthermore the superior energy resolution allows for in-situ background identification. The background modeling is based on the observed $\gamma$ lines and not only on the material screening results. Due to these facts a germanium experiment has the highest discovery potential among all experiments with similar sensitivity even when operating with less mass.

\subsection{GeRda: Phase I}

GERDA [3] is located in the Hall A of the LNGS underground laboratory of INFN in Italy. The main part of the experimental setup consist of a $64 \mathrm{~m}^{3}$ liquid argon cryostat placed in a water tank filled with $590 \mathrm{~m}^{3}$ purified water. The water provides additional shielding and is acting as Cerenkov veto against muons. The construction of the experiment was completed in November 2009 and the cryostat was filled with liquid argon in the same year.

The main difference from the previous experiments is that in GERDA the HPGe detectors are operated directly in a cryogenic liquid (liquid argon). The advantage being that the cooling liquid acts as shielding against external radiation.

In Phase I of the experiment enriched germanium detectors from the Heidelberg-Moscow and IGEX experiments were used. Phase I was completed reaching an exposure of about $21 \mathrm{~kg} \cdot \mathrm{yr}$ with a background level of $10^{-2} \mathrm{cts} /(\mathrm{keV} \cdot \mathrm{kg} \cdot \mathrm{yr})$. GERDA Phase I set a limit on the $0 v \beta \beta$ decay of ${ }^{76} \mathrm{Ge}$ of $T_{1 / 2}^{0 v}>2.1 \cdot 10^{25} \mathrm{yr}[4]$.

\section{Phase II upgrade}

In parallel with Phase I the collaboration pursued a detector production project for Phase II. From the available $37.5 \mathrm{~kg}$ newly procured enriched material, 30, Broad Energy Germanium (BEGe) type detectors were produced five of which were already deployed during Phase I.

Since the background in Phase I can be explained entirely with nearby sources [5] a considerable effort was made to reduce the activity of the surrounding materials. The detector holders underwent a major upgrade replacing most of the copper parts with semiconductor grade crystalline silicon. Flat cables are taking the signal to the cryogenic amplifiers located about $30 \mathrm{~cm}$ 
above the detector array. The amplifiers are based on the same concept as in Phase I with an improved design. The contact from the cable to the detector is realized with wedge bonding for better electrical contact and for mass reduction.
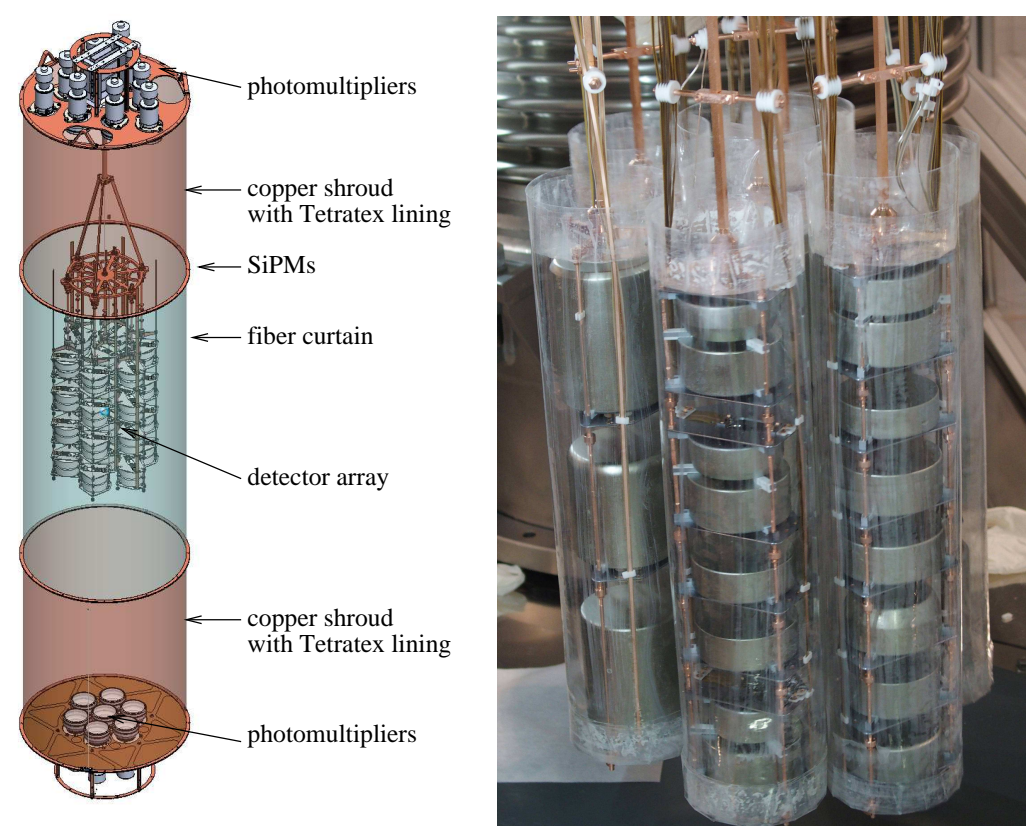

Figure 1: Drawing of the Phase II setup with LAr-veto. Right: the seven detector strings before deployment.

A major hardware upgrade was necessary to accommodate light instrumentation in the GERDA cryostat. The new Phase II lock is a larger version of the one used in Phase I with an internal diameter of $500 \mathrm{~mm}$ and a height of about $2.5 \mathrm{~m}$.

A design criteria for the LAr-veto was the possibility to be deployed together with the HPGe detectors. Other important requirements were reduced weight and negligible contribution to the background. The decision finally fell on a hybrid system comprising photo-multipliers (PMTs) and wave-length shifting (WLS) fibers equipped with silicon photo-multipliers (SiPMs).

The LAr-veto system is a cylindrical object that fits inside the Phase II lock and is built around the HPGe array (see Fig.1). PMTs are mounted on the top and bottom plates of the setup, 16 in total. The thin walled copper cylinders in between are lined with Tetratex reflector material coated with Tetraphenyl-butadiene (TPB).

The middle section of the LAr veto consists of a dense curtain of WLS fibers. The fibers are coated with TPB by vacuum deposition to make them sensitive to the VUV light. Both ends of the fibers are coupled to SiPMs, nine fibers to one $3 \mathrm{~mm}$ x $3 \mathrm{~mm}$ SiPM from Ketek GmBH. The fibers collect light from both inside and outside of the enclosed volume.

In Phase I the HPGe arrays were encapsulated in hermetically closed thin walled copper cylinders to prevent ${ }^{42} \mathrm{~K}$ ions migrating to the detector surface and decay there. In Phase II the copper shrouds were replaced with ones made of transparent nylon allowing the scintillation light produced close to the detectors to reach the LAr-veto. The nylon is coated with polystyrene-TPB solution. 


\section{Phase II LAr-veto commissioning}

Prior to the start of Phase II data taking the LAr veto was tested with one detector string and various calibration sources.

In the data recorded with a ${ }^{228} \mathrm{Th}$ source the Compton continuum around $2040 \mathrm{keV}$ is suppressed by about a factor 100 just with the LAr-veto alone (Fig.3). When Pulse Shape Discrimination (PSD) is also applied the total suppression is about 300 fold.

The effect of the LAr-veto can be also studied in-situ using the physics data. The $1525 \mathrm{keV}$ line of ${ }^{42} \mathrm{~K}$ (daughter of ${ }^{42} \mathrm{Ar}$ ) is suppressed by about a factor of 5 due to the coincident beta decay. On the other hand ${ }^{40} \mathrm{~K}$ present in the surrounding material emits a single $\gamma$ photon with a branching ratio of $10.7 \%$ so the $1460 \mathrm{keV}$ line cannot be suppressed by the LAr-veto. Hence the suppression of the ${ }^{40} \mathrm{~K}$ line is a measure of the dead time introduced by the LAr-veto which was found to be $2.3 \%$.
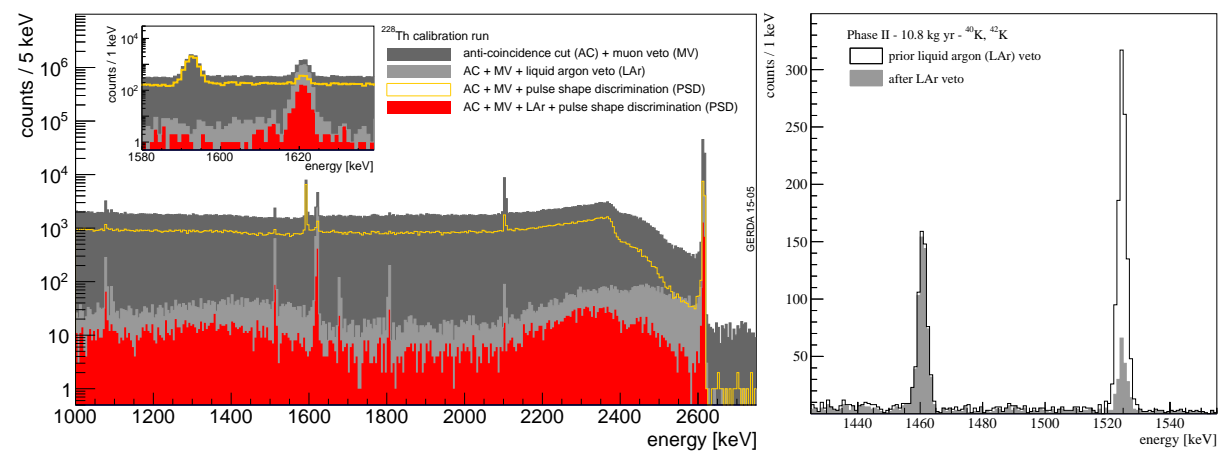

Figure 2: Right: ${ }^{228} \mathrm{Th}$ spectrum suppressed by LAr-veto and PSD. Right: effect of the LAr-veto on ${ }^{40} \mathrm{~K}$ and ${ }^{42} \mathrm{~K}$ lines in the physics data.

\section{Phase II: first results}

After a stable configuration was reached with 40 detectors arranged in 7 strings GERDA has started Phase II on December 20, 2015. After this date the data were blinded in a window of $Q_{\beta \beta} \pm$ $25 \mathrm{keV}$.

To assure the energy scale stability weakly calibration runs were performed with ${ }^{228} \mathrm{Th}$ sources. The resolution of the detectors at $2.6 \mathrm{MeV}$ was $3.2 \mathrm{keV}$ FWHM for the BEGe detectors and 3.8 keV FWHM for the coaxial detectors.

It is remarkable that after applying the LAr-veto to the physics data an almost pure sample of $2 v \beta \beta$ decays is left with a count rate that corresponds to the half life measured in Phase I (see Fig. 3 left). In the right plot of Fig. 3 the effect of the PSD cut is demonstrated on physics data. The ratio of the peak amplitude of the current signal and the total energy is used as a single parameter cut for PSD in BEGe detectors (A/E cut). A low value of the A/E parameter is typical for multi-site events and a high value for surface events. Only a narrow band of signal like events passes the cut. The exact value of the cut parameter was optimized in-situ for each detector using calibration data. 
After all analysis cuts were tuned on the blinded data the first unblinding of Phase II data happened in June 2016 when $10.8 \mathrm{~kg} \cdot \mathrm{yr}$ exposure was reached. In addition a remaining of 1.9 $\mathrm{kg}$.yr Phase I data recorded after May 2013 was also unblinded and analyzed. Fig.4 shows the Phase II spectra of the enriched coaxial and BEGe detectors.

An important result of Phase II is the achieved background level. In a window of $Q_{\beta \beta} \pm 100$ $\mathrm{keV}$ the number of counts in the coaxial data set corresponds to $3.5_{-1.5}^{+2.5} \cdot 10^{-3} \mathrm{cts} /(\mathrm{keV} \cdot \mathrm{kg} \cdot \mathrm{yr})$ and in the BEGe data set $0.7_{-0.5}^{+1.1} \cdot 10^{-3} \mathrm{cts} /(\mathrm{keV} \cdot \mathrm{kg} \cdot \mathrm{yr})$.

A combined fit was performed using the published Phase I data [4] and the $12.7 \mathrm{~kg} \cdot \mathrm{yr}$ unblinded data (see Table 1). The region of interest with the combined fit is shown in Fig.5. The fit resulted in a half life limit of: $T_{1 / 2}>5.3 \cdot 10^{25} \mathrm{yr}$. Assuming no signal the median sensitivity of the experiment is $4.0 \cdot 10^{25} \mathrm{yr}(90 \% \mathrm{CL})$.
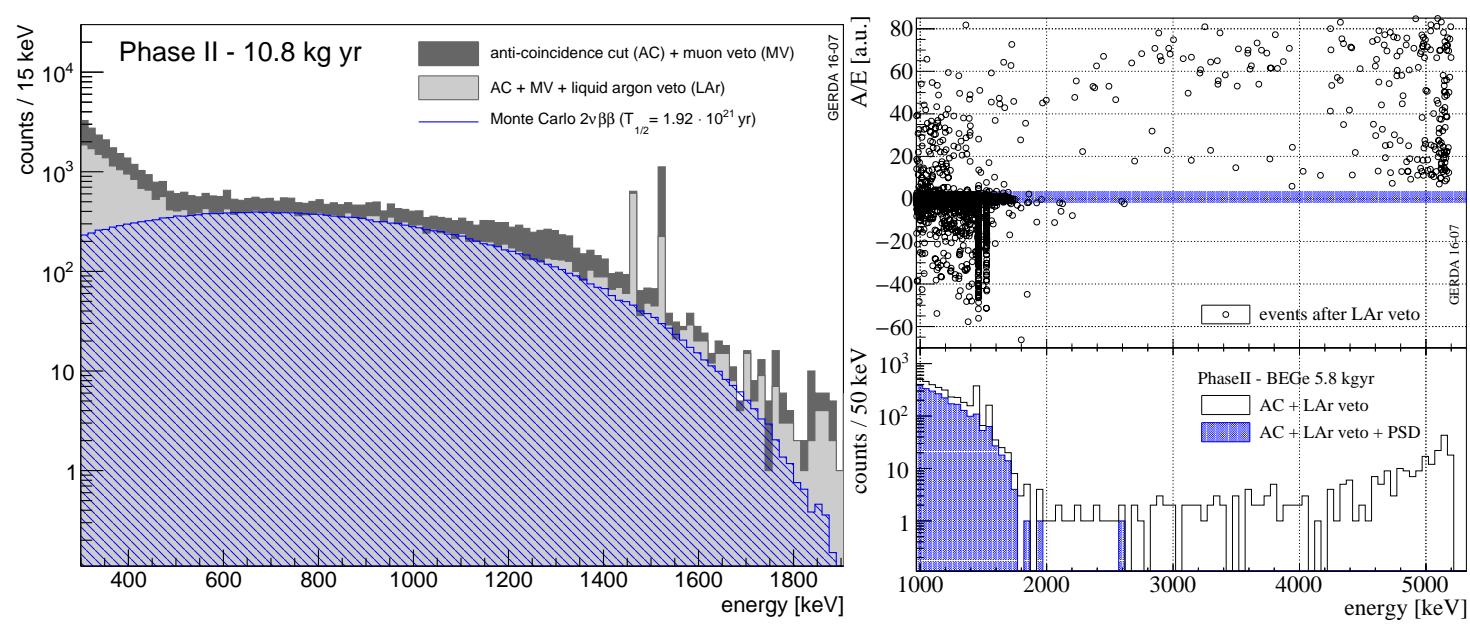

Figure 3: Left: after applying the LAr veto in the low energy part of the spectrum an almost pure $2 v \beta \beta$ remains. The blue shaded area is the simulated $2 v \beta \beta$ spectrum scaled to the half life measured in Phase I. Right: The PSD cut applied to the BEGe physics data after the LAr veto. In the signal band of the A/E parameter only one event survives all cuts in $Q_{\beta \beta} \pm 100 \mathrm{keV}$
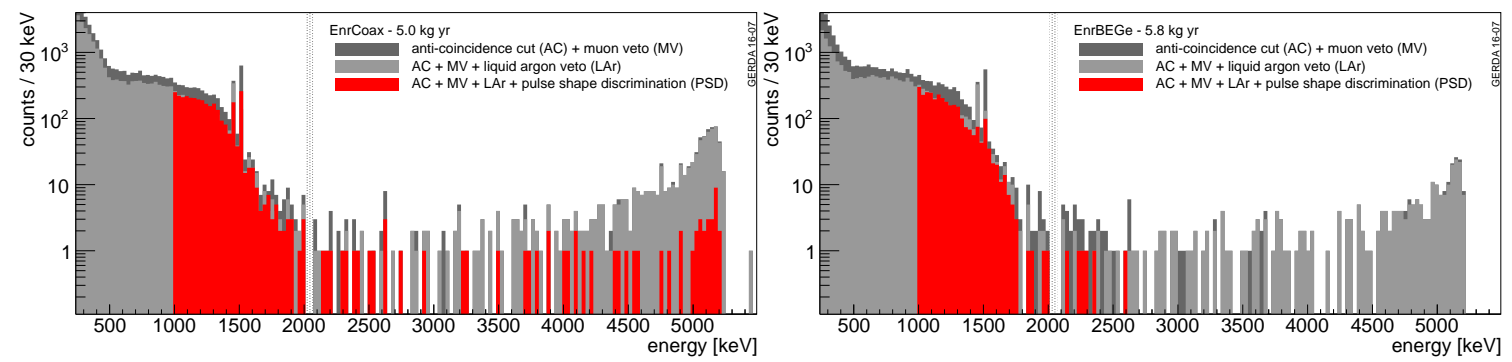

Figure 4: Phase II results. Left: the full spectrum of coaxial detectors. Right: the spectrum of BEGe detectors. 


\begin{tabular}{c|c|c|c|c} 
data set & exposure $[\mathrm{kg} \cdot \mathrm{yr}]$ & FWHM [keV] & efficiency & background $\left[10^{-3} \mathrm{cts} /(\mathrm{keV} \cdot \mathrm{kg} \cdot \mathrm{yr})\right]$ \\
\hline PI golden & 17.9 & $4.27 \pm 0.13$ & $0.57 \pm 0.03$ & $11 \pm 2$ \\
PI silver & 1.3 & $4.27 \pm 0.13$ & $0.57 \pm 0.03$ & $30 \pm 10$ \\
PI BEGe & 2.4 & $2.74 \pm 0.20$ & $0.66 \pm 0.02$ & $5_{-3}^{+4}$ \\
PI extra & 1.9 & $4.17 \pm 0.19$ & $0.58 \pm 0.04$ & $5_{-2}^{+4}$ \\
\hline PII coax & 5.0 & $4.0 \pm 0.2$ & $0.53 \pm 0.05$ & $3.5_{-2}^{+2}$ \\
PII BEGe & 5.8 & $3.0 \pm 0.2$ & $0.60 \pm 0.02$ & $0.7_{-0.5}^{+1.1}$ \\
\hline
\end{tabular}

Table 1: Data sets used for the half-life analysis including the background level and the signal efficiencies with all cuts applied.
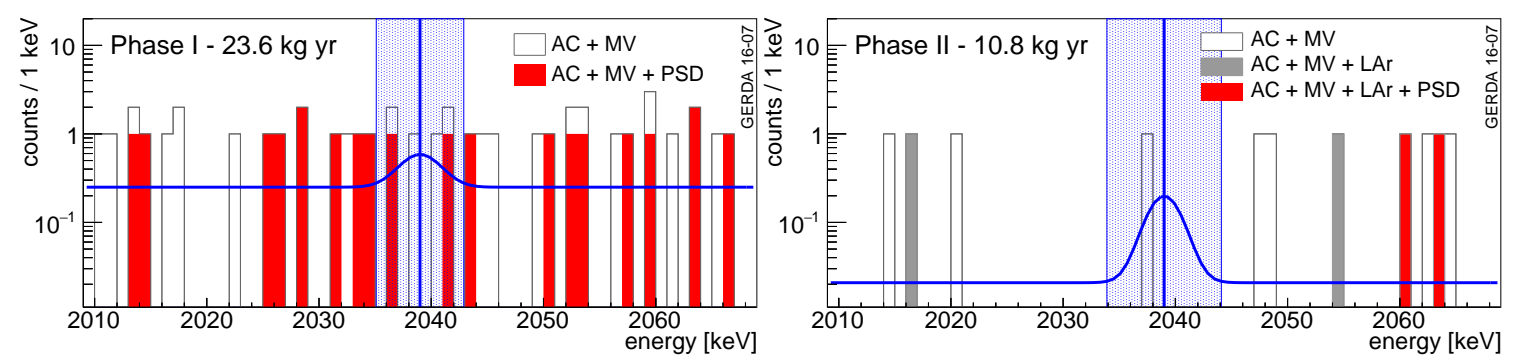

Figure 5: Zoom on the region of interest. Left: Phase I data, right: Phase II data. The blue shaded area shows the region of interest: $Q_{\beta \beta} \pm 3 \sigma$.

\section{Summary}

GERDA Phase II currently is taking data with $35.8 \mathrm{~kg}$ of enriched germanium detectors. The design goal of Phase II was accomplished. The experiment is running practically background free. The background level in the BEGe data set corresponds to $0.7 \cdot 10^{-3} \mathrm{cts} /(\mathrm{keV} \cdot \mathrm{kg} \cdot \mathrm{yr})$. With this achievement GERDA has the lowest background level among all running $0 v \beta \beta$ experiments when expressed in number of counts in the region of interest.

In addition to the Phase II data we unblinded a data set with $1.9 \mathrm{~kg} \cdot \mathrm{yr}$ exposure from Phase I that was recorded after the Phase I data release in May 2013 [4]. A new half life limit for the $0 v \beta \beta$ decay was computed using Phase I and Phase II data combined. The analysis gave $T_{1 / 2}>5.3 \cdot 10^{25} \mathrm{yr}$ while the calculated median sensitivity of the experiment is $4 \cdot 10^{25} \mathrm{yr}(90 \%$ CL).

\section{References}

[1] S. F. Ge, M. Lindner and S. Patra, arXiv:1508.07286

[2] J. C. Helo and M. Hirsch, arXiv:1509.00423

[3] K.-H. Ackermann et al., Eur. Phys. J. C, 73, 3, 2013

[4] Agostini, M. et al., Phys. Rev. Lett., 111, 12, 2013

[5] Agostini, M. et al., Eur. Phys. J. C, 74, 2764, 2014 